\title{
The challenge of developing human 3D organoids into medicines
}

\author{
Joaquim Vives ${ }^{1,2,3^{*}}$ (D) and Laura Batlle-Morera ${ }^{4^{*}}$
}

\begin{abstract}
The capacity of organoids to generate complex 3D structures resembling organs is revolutionizing the fields of developmental and stem cell biology. We are currently establishing the foundations for translational applications of organoids such as drug screening, personalized medicine and launching the future of cell therapy using organoids. However, clinical translation of organoids into cell replacement therapies is halted due to (A) a few preclinical studies demonstrating their efficacy and (B) the lack of robust, reproducible, and scalable methods of production in compliance with current pharmaceutical standards. In this issue of Stem Cell Research \& Therapy [ref], Dossena and collaborators present a validated bioprocess design for large-scale production of human pancreatic organoids from cadaveric tissue in accordance with current good manufacturing practice. The authors also propose a set of specifications of starting materials and critical quality attributes of final products that are of interest to other developments provided that this type of medicines are different than any other medicinal product due to their complex composition and living nature of the active ingredient. Although large-scale production of functional cells secreting insulin is still a challenge, the development of methods such as the one presented by Dossena and collaborators contributes to move toward clinical use of organoids in the treatment of type 1 diabetes and opens avenues for future clinical use of organoids in degenerative pathologies.
\end{abstract}

Keywords: Good manufacturing practice, Organoid, Pancreas, Regenerative medicine, Tissue engineering, Regulatory and quality compliance

\section{Background}

Organoids are three-dimensional (3D) cell structures grown in vitro from stem cells, primarily isolated either from biopsies or from pluripotent stem cells, that recapitulate key features of both the development and performance of native organs [1]. Cells forming organoids are able to differentiate, undergo lineage commitment, and acquire the specific tissue patterning to develop into several endoderm, mesoderm, and ectoderm-derived tissues, mimicking somehow their in vivo counterparts. The resulting structures are complex and provide a unique opportunity to model human organ development and disease in a system remarkably similar to that occurring in vivo.

Despite the extensive use of organoids in basic research, their translational biomedical application is currently

\footnotetext{
* Correspondence: jvives@bst.cat; Laura.Batlle@crg.eu

${ }^{1}$ Cell Therapy Service, Blood and Tissue Bank (BST), Barcelona, Catalonia, Spain

${ }^{4}$ Core Facilities Program, Centre for Genomic Regulation (CRG), Barcelona, Catalonia, Spain

Full list of author information is available at the end of the article
}

restricted to drug testing $[2,3]$ and initial cell replacement strategies $[4,5]$. However, the regulatory framework does not define specific guides or rules to such type of entities and, for instance, drug testing using organoids in Europe needs be done in accordance with a very generic "Guideline on the principles of regulatory acceptance of 3Rs (replacement, reduction, refinement) testing approaches" (EMA/ CHMP/CVMP/JEG-3Rs/450091/2012) by the European Medicines Agency (EMA). Currently, the clinical use of organoids would need to meet the requirements for cell and gene-based therapies and produced the following good manufacturing practices (GMP) as any other pharmaceutical drug [6].

In any case, organoids hold a huge potential in regenerative medicine purposes if current limitations are overcome, namely, (A) clonal variation, developmental stage, pluri-/multi-potency, and chromosomal stability of stem cells; (B) reproducibility, accuracy, and scalability of the methodologies proposed; (C) meaningful functional assessment of resulting organoids; and (D) preclinical 
validation [7]. Only if these constraints are conveniently addressed, organoid technology will be readily translated into novel approaches for the replacement of severely damaged organs, such as the pancreas in patients with type 1 diabetes for instance. Indeed, the generation of human pancreas organoids (hPOs) in compliance with pharmaceutical standards is a challenge in view that currents methods are laborious, time-consuming, expensive and, as stated previously, lack reproducibility [8]. In this context, improvements in bioprocess design and definition of strategies in compliance with quality and regulatory guidelines are needed, being crucial the use of chemically defined serum-free culture media and the establishment of robust specifications of starting materials and the critical quality attributes (CQA) of final product $[9,10]$. This can only be understood considering hPOs as a cell-based medicinal product, from a pharmaceutical production perspective, in compliance with current GMP guidelines.

\section{Quality compliance in the production of organoids}

GMP in cell therapy refers to a system for ensuring that medicinal products are consistently manufactured and controlled according to quality standards, thus minimizing the risks involved in the production process that cannot be eliminated otherwise through testing the final drug product, so they are safe to be used in patients. To achieve this, GMP covers all aspects of production (e.g., starting materials, facilities, and equipment), personnel (e.g., training, hygiene of staff), and documentation (e.g., standard operating procedures and registers to prove that they are consistently followed at each step in the manufacturing process) [6]. In fact, protocols for the development of human-induced pluripotent stem cell (iPSC)-derived retinal organoids have been successfully adapted to GMP standards required for the generation of transplantable photoreceptor cells for future clinical applications [11]. Similarly, Takebe and collaborators have further developed their initial liver buds protocol for massive production and reproducibility making it ready for further GMP adaptation in order to scale up production in GMP facilities for transplantation purposes [12]. Moroever, the production of cells for human application greatly benefits from the use of fully defined, xeno-free conditions rather than media supplemented with sera, thus improving reproducibility and preventing xeno-mediated infection or immune rejection when transplanted into patients.

The generation of hPOs from the human pancreas is feasible, although not at the scale necessary to result in sufficient number of cells for clinical use [13]. In their recent paper, Dossena and collaborators fixed a series of in-process controls and improved media formulation by replacing R-spondin-1 (RSPO1) conditioned medium $(\mathrm{CM})$ with recombinant RSPO1 protein, resulting in a GMP-compliant protocol intended to deliver a cryopreserved hPO for further use in allogeneic therapies for type 1 diabetes [14].

Similar to other cell-based therapies, Dossena and collaborators paid special attention to define the criteria of acceptance of the starting materials in order to minimize the risk of transmittable viral diseases and to ensure the

Table 1 Quality product profile for hPO. Critical quality attributes of hPO and acceptance proposed by Dossena and collaborators that can be adapted in other developments of organoid production in accordance with pharmaceutical quality standards. Additional controls may include determination of endotoxins, mycoplasma, and sterility test according to the pharmacopeia. Ab antibody, Ag antigen, HBC hepatitis B core, HBV hepatitis B virus, HCV hepatitis C virus, HIV human immunodeficiency virus, VDRL-TPHA venereal disease research laboratory test-treponema pallidum hemagglutination test

\begin{tabular}{ll}
\hline In process controls & Acceptance criteria \\
\hline Donor & Negative \\
Serology (HIV, HBV, HCV, anti-HBC, VDRL-TPHA) & Absence \\
Risk factors, systemic infections, diabetes & Within 3 times the normal range \\
Amylase levels & \\
Biopsy & $21 \pm 4^{\circ} \mathrm{C}$ \\
Transport & \\
hPO & Within $36 \mathrm{~h}$ from biopsy \\
Isolation & Presence of ductal progenitors (PDX $1^{+} / S O X 9^{+}$) \\
Identity (using ductal, mesenchymal, hematopoietic and endothelial markers) & Normal glucose consumption and lactate production \\
Metabolism & $\geq 70 \%$ \\
Viability & Capacity to differentiate into insulin-secreting cells \\
Potency & Sterile \\
Sterility (BacTAlerT) & Normal \\
Karyotype &
\end{tabular}


procurement of healthy pancreatic tissue for the production of hPO (Table 1). To do so, pancreas biopsies are processed within $36 \mathrm{~h}$ from extraction from donors aged 18-65 years, beating heart and declared brain death, known cause of death, negative for HIV, HBV, HCV, anti-HBc, VDRL-TPHA, absence of risk factors, systemic infections, and diabetes, amylase levels within three times the normal range [14]. Similar to the original small-scale research method, the GMP-validated production process resulted in hPOs with equivalent dimensions, growth kinetics, metabolism, and cellular composition. In the characterization of hPOs, the authors reported the presence of ductal cells and ductal progenitors but differentiation into endocrine cells producing insulin was not demonstrated in this work. Remarkably, viability rates were always above $75 \%$ after thawing thus demonstrating the feasibility of generating a bank of cryopreserved organoids, which could be further differentiated, either in vitro or in vivo, into insulin-producing cells, as already shown to be feasible by other authors [15]. This is a key point that will need to be addressed next to ensure clinical success, provided that the potency of hPO generated in this work will rely on their capacity to generate insulin and restore normoglycemia.

\section{Outlook}

Although further research is required in order to evaluate the therapeutic benefit of organoids in the context of diabetes or other pathologies, only the adherence to strict quality standards will ensure reproducibility. The efficacy and safety of organoid-based therapies will require appropriate cellular composition, proper engraftment and vascularization into the host and the demonstration of functional activity. Moreover, translation of organoid technologies into the clinics may benefit from the emergence of human leukocyte antigen (HLA)homozygous iPSC initiatives, which hold the potential to foster the development of patient compatible regenerative therapeutic approaches that can embrace large-scale production of organoids and organoids biobanking [16]. Furthermore, the blending of organoid technology with 3D bioprinting and vascularization approaches may lead to macrostructures with the desired cellular composition for successful transplantation [17]. The combination of all these technologies is key for realizing the full potential of organoids as therapies.

\section{Abbreviations}

3D: Three-dimensional; CM: Conditioned media.; CQA: Critical quality attributes; CRISPR: Clustered regularly interspaced short palindromic repeats; EMA: European Medicines Agency; GMP: Good manufacturing practice; HLA: Human leukocyte antigen; hPO: Human pancreas organoid; iPSC: Induced pluripotent stem cell
Authors' contributions

All authors wrote the manuscript. All authors revised the manuscript. Both authors approved the final version of the manuscript.

\section{Funding}

Work in JV's laboratory is supported by the Spanish Cell Therapy Network (TerCel, expedient no. RD16/0011/0028), awarded by Generalitat de Catalunya as Consolidated Research Group (ref. 2017-SGR-719). Project PI19/ 01788 is funded by Instituto de Salud Carlos III and co-funded by European Union (ERDF/ESF) - A way to build Europe.

\section{Availability of data and materials}

Not applicable.

Ethics approval and consent to participate

Not applicable.

Consent for publication

Not applicable.

\section{Competing interests}

The authors declare that they have no competing interests.

\section{Author details}

${ }^{1}$ Cell Therapy Service, Blood and Tissue Bank (BST), Barcelona, Catalonia, Spain. ${ }^{2}$ Musculoskeletal Tissue Engineering Group, Vall d'Hebron Research Institute (VHIR), Universitat Autònoma de Barcelona (UAB), Barcelona, Catalonia, Spain. ${ }^{3}$ Medicine Department, Universitat Autònoma de Barcelona (UAB), Barcelona, Catalonia, Spain. ${ }^{4}$ Core Facilities Program, Centre for Genomic Regulation (CRG), Barcelona, Catalonia, Spain.

Received: 26 December 2019 Revised: 26 December 2019 Accepted: 4 February 2020

References

1. Clevers H. Modeling development and disease with organoids. Cell. 2016; 165(7):1586-97.

2. Dekkers JF, Berkers G, Kruisselbrink E, Vonk A, de Jonge HR, Janssens HM, et al. Characterizing responses to CFTR-modulating drugs using rectal organoids derived from subjects with cystic fibrosis. Sci Transl Med. 2016; 8(344):344ra84.

3. Takahashi T. Organoids for drug discovery and personalized medicine. Annu Rev Pharmacol Toxicol. 2019;59:447-62.

4. Takebe T, Sekine K, Enomura M, Koike H, Kimura M, Ogaeri T, et al. Vascularized and functional human liver from an iPSC-derived organ bud transplant. Nature. 2013;499(7459):481-4.

5. Sugimoto S, Ohta Y, Fujii M, Matano M, Shimokawa M, Nanki K, et al. Reconstruction of the human colon epithelium in vivo. Cell Stem Cell. 2018; 22(2):171-6.e5.

6. Vives J, Carmona G, Vives J, Carmona G. Guide to Cell Therapy GxP. 1st ed. London: Academic Press (Elsevier); 2015. p. 266.

7. Pacitti D, Privolizzi R, Bax BE. Organs to cells and cells to organoids: the evolution of. Front Cell Neurosci. 2019;13:129.

8. Dayem AA, Lee SB, Kim K, Lim KM, Jeon TI, Cho SG. Recent advances in organoid culture for insulin production and diabetes therapy: methods and challenges. BMB Rep. 2019;52(5):295-303.

9. Fischbach MA, Bluestone JA, Lim WA. Cell-based therapeutics: the next pillar of medicine. Sci Transl Med. 2013:5(179):179ps7.

10. Schneider CK, Salmikangas P, Jilma B, Flamion B, Todorova LR, Paphitou A, et al. Challenges with advanced therapy medicinal products and how to meet them. Nat Rev Drug Discov. 2010;9(3):195-201.

11. Gagliardi G, Ben M'Barek K, Chaffiol A, Slembrouck-Brec A, Conart JB, Nanteau C, et al. Characterization and transplantation of CD73-positive photoreceptors isolated from human iPSC-derived retinal organoids. Stem Cell Rep. 2018;11(3):665-80.

12. Takebe T, Sekine K, Kimura M, Yoshizawa E, Ayano S, Koido M, et al. Massive and reproducible production of liver buds entirely from human pluripotent stem cells. Cell Rep. 2017;21(10):2661-70.

13. Broutier L, Andersson-Rolf A, Hindley CJ, Boj SF, Clevers H, Koo BK, et al. Culture and establishment of self-renewing human and mouse adult liver 
and pancreas 3D organoids and their genetic manipulation. Nat Protoc. 2016;11(9):1724-43.

14. Dossena M, Piras R, Cherubini A, Barilani M, Dugnani E, Salanitro F, et al. Standardized GMP-compliant scalableproduction of human pancreas organoids. Stem Cell Res Ther. https://doi.org/10.1186/s13287-020-1585-2.

15. Loomans CJM, Williams Giuliani N, Balak J, Ringnalda F, van Gurp L, Huch M, et al. Expansion of adult human pancreatic tissue yields organoids harboring progenitor cells with endocrine differentiation potential. Stem Cell Rep. 2018;10(3):712-24

16. Alvarez-Palomo B, Vives J, Casaroli-Marano RPP, G Gomez SG, Rodriguez Gómez L, Edel MJ, et al. Adapting cord blood collection and banking standard operating procedures for HLA-homozygous induced pluripotent stem cells production and banking for clinical application. J Clin Med. 2019; 8(4):476.

17. Takebe T, Wells JM. Organoids by design. Science. 2019;364(6444):956-9.

\section{Publisher's Note}

Springer Nature remains neutral with regard to jurisdictional claims in published maps and institutional affiliations. 